\title{
GESTIÓN DEL CAPITAL INTELECTUAL EN CARRERAS DE INGENIERÍA PARA EL DESARROLLO TECNOLÓGICO EN
}

\author{
LA INDUSTRIA PERUANA
}

\author{
INTELLECTUAL CAPITAL MANAGEMENT IN \\ ENGINEERING CAREERS FOR TECHNOLOGICAL \\ DEVElopment in THE PERUVIAN INDUSTRY
}

\author{
Fernando Ormachea Freyre y Erik Papa Quiroz \\ Universidad Nacional Mayor de San Marcos, Unidad de Posgrado de Ingeniería de Sistemas e \\ INFORMÁTICA
}

\begin{abstract}
RESUMEN
El presente artículo presenta una propuesta de rediseño de la gestión de prácticas preprofesionales, integrando unidades académicas y de investigación con docentes y alumnos, para que interactúen en investigaciones aplicadas bajo el esquema de comunidades de práctica planificadas, apoyados por tecnologías de información y comunicación que faciliten la comunicación directa o a distancia, la captura, el procesamiento y la consolidación de conocimiento disperso, generando capital intelectual de aplicación útil para el beneficio de las empresas y de las carreras involucradas.
\end{abstract}

Descriptores: capital intelectual, comunidad de práctica, aprendizaje colaborativo

\begin{abstract}
This article presents a proposal for redesigning the management of internships by integrating academic and research units, with teachers and students interacting in applied research under the scheme of planned communities of practice, supported by information and communication technologies to facilitate direct or remote communication, capture, processing and consolidation of dispersed knowledge, generating intellectual capital useful for the benefit of businesses and careers involved.
\end{abstract}

Keywords: intellectual capital, community of practice, collaborative learning

\section{INTRODUCCIÓN}

La gestión del conocimiento permite obtener capital intelectual, es decir, conocimiento explícito aplicado, logrando tanto la mejora de capacidades como de productividad.

No se ha llegado a tal efectivo desarrollo por la carencia de lo que Cohen (1990) definió como capacidad de absorción del conocimiento, facultad que sí está presente en las grandes empresas. Las universidades, a través de investigaciones, generan conocimiento, pero, en general:

- Es intermitente, no permanente;

- Integra limitadamente a docentes, alumnos y egresados y se carece de recurso humano;

- No llega a generar cultura investigadora; y

- No enfatiza la generación de capital intelectual replicable y de impacto. 
Por lo anterior, se requiere potenciar la gestión del conocimiento desde las actividades de pregrado, que integre a docentes, egresados y alumnos y planifique investigaciones aplicadas y básicas de mayor alcance, con un soporte tecnológico apropiado para implantar librerías digitales y redes interinstitucionales donde se comparta y colabore y conforme "comunidades de práctica normales y virtuales" tuteladas por las universidades.

Bajo esta situación, el presente artículo mostrará las evidencias de oportunidades y un camino factible para potenciar y reenfocar el conocimiento generado en los programas académicos de pregrado hacia aplicaciones de tipo horizontal y de amplia cobertura, contribuyendo a la mejora tecnológica de nuestra industria.

Como preámbulo, se presentan y discuten cuatro aportes sobre la problemática planteada, basados en la participación sustantiva de los centros formativos.

\section{El modelo Intellectus}

La gestión del conocimiento en las universidades es abordada sucintamente por Bueno et al. [2]. Su modelo considera el proceso completo de generación de conocimiento y capital intelectual, destacando el acertado enfoque de utilización del capital humano, estructural y relacional, para lograr resultados mensurables, en base al capital intelectual generado, las actividades de investigación y las redes generadas. Considera, además, el proceso de benchmarking, pero enfatiza la producción científica, mas no tanto el capital intelectual desde el punto de vista de la industria.

La aplicación del capital intelectual generado y aplicado en la industria constituye un indicador trascendental para medir el desarrollo tecnológico en la industria vía el aporte de una universidad. Por tanto, un aporte al modelo consiste en que, además de medir la producción de tipo científico, mida la aplicabilidad efectiva de lo aplicado en la industria.

\section{Las universidades como agentes para difundir capital intelectual}

García [3] plantea la siguiente base:
Se puede afirmar que la gestión del conocimiento dentro de la relación universidad-mundo productivo, tiene como objetivo fundamental darle aplicabilidad e incorporarle valor agregado al conocimiento generado tanto en la academia como en la empresa, para así convertirlo en capital intelectual, el cual es un recurso intangible que le permitirá a la articulación adaptarse eficientemente a las transformaciones del entorno $y$ entrar a la sociedad del conocimiento.

Destacamos su tercer objetivo:

Crear el ambiente institucional adecuado para la realización de actividades de innovación y desarrollo tecnológico: donde se forme y estimule al estudiante para desarrollar investigación en su disciplina y para participar en proyectos concretos desde el comienzo y durante toda su carrera universitaria.

En nuestra investigación hemos detectado que se puede potenciar lo anterior, con lo siguiente:

- Partir de estudios sectoriales;

- Enfocar la gestión de tipo colectivo;

- Aprovechar las TIC;

- Implantar comunidades de práctica; e

- Implantar programas de prácticas pre profesionales orientadas por docentes, logrando capital intelectual a través del trabajo de campo y bajo entorno colaborativo.

\section{Las comunidades de práctica en las universidades}

Warren [4] documenta experiencias de comunidades de práctica tuteladas dentro del ámbito académico y propone lo siguiente: “... el desafío es permitir a los estudiantes obrar recíprocamente con la comunidad de práctica de una manera significativa ...". Y perfila con suficiencia el concepto general de las comunidades de práctica $(\mathrm{CoP})$ dentro del ámbito universitario. Cabe precisar que Warren enfoca a las $\mathrm{CoP}$ orientadas al emprendimiento, comprensible por tratarse del Reino Unido.

En el Perú, necesitamos una etapa previa: sentar bases firmes de conocimientos técnicos básicos que aseguren la efectividad tecnológica de la industria y que a partir de ello se pueda emprender modelos de mayor desarrollo y apoyados por empresas inversionistas. 


\section{Gestión del conocimiento en un departamento universitario}

Ditzel [5] presenta un caso aplicativo en pregrado de una facultad de Matemáticas, mostrando ventajas del trabajo colaborativo y su articulación con otras facultades. Presenta su modelo cíclico con tres procesos fundamentales de la gestión del conocimiento: el desarrollo, la transferencia y la utilización; contando con el soporte de otros cuatro: recursos humanos, contacto y comunicación, calificación académica y proceso informático.

Asimismo, muestra acertadamente la necesidad de implantar un soporte informático basado en tecnología web con arquitectura de portal, y definir una taxonomía para el conocimiento a almacenar y relacionar.

Observamos un enfoque tipo "push", es decir, a partir de los desarrollos internos se lograría, en un futuro, el aporte hacia la industria.

Nuestro modelo plantea un enfoque tipo "pull", esto es, a través de los requerimientos actuales se establecerían los objetivos, prioridades y planes de investigación básica y aplicada para organizar los equipos de investigación y desarrollar el capital intelectual que ya necesiten las empresas.

Basándonos en los antecedentes, las discusiones planteadas en cada caso y nuestras pruebas piloto, presentamos los planteamientos para nuestra propuesta dirigida al segmento de las pequeñas y medianas empresas (PYMES), que cuenta con menos recursos y mayor necesidad de apoyo para desarrollarse:

\section{Problema en el ámbito académico:}

Falta de sinergia y gestión integrada de la investigación aplicada con la gestión del capital humano, estructural y relacional que ya se poseen.

II. Problema en el ámbito de la gestión tecnológica de los procesos en las PYMES:

Falta de capital humano y estructural en cantidad y calidad, que pueda aportar al desarrollo tecnológico.
III. Objetivo de la investigación:

Diseñar un modelo de gestión del capital intelectual en las carreras universitarias de ingeniería que integre y aproveche los recursos en pregrado.

\section{Hipótesis principal:}

Integrando el proceso de prácticas preprofesionales con el de investigación aplicada, en las carreras de ingeniería, se produce capital intelectual (CI).

Nuestra propuesta se basará en complementar el aporte normal de las facultades de Ingeniería hacia las PYMES, potenciando el capital estructural, el capital relacional y el capital humano, y organizando comunidades de práctica conformadas por docentes, practicantes y especialistas.

\section{Procedimientos de investigación}

Se desarrollaron las siguientes actividades:

1. Entrevistas a docentes e investigadores para identificar factores relevantes para el modelo;

2. Selección de la carrera de ingeniería;

3. Encuestas a universidades sobre el potencial de actividad investigadora en pregrado;

4. Contactos, documentación de experiencias y selección de la universidad para los pilotos;

5. Recopilación de información básica de la gestión y procesos de actual ejecución;

6. Encuestas a practicantes y a jefes de los practicantes en empresas;

7. Identificación de elementos organizacionales para el modelo;

8. Formulación del modelo;

9. Identificación de los flujos de capital;

10. Conformación de una comunidad de práctica; y

11. Evaluación del potencial de capital intelectual generable y requerimientos organizacionales y de sistemas.

\section{RESULTADOS Y DISCUSIÓN}

Sobre las encuestas a universidades: Se obtuvo información de 10 facultades o unidades de Ingeniería Industrial (de un total de 30). Se realizaron cinco grupos de preguntas, de las cuales presentamos el siguiente resultado representativo: 


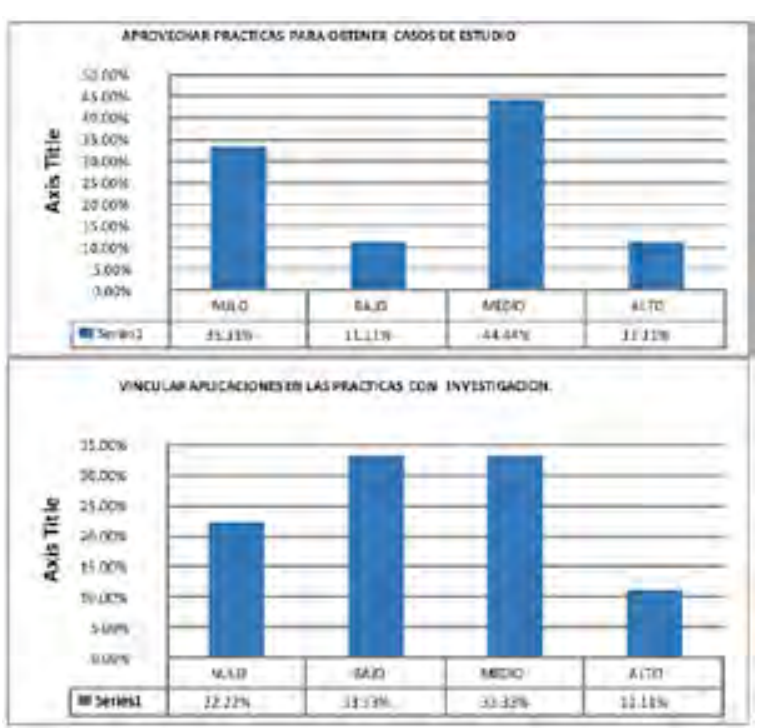

Figura 1: Expectativas actuales sobre el potencial académico de las prácticas.

Considerando sólo las expectativas de orden medio $\mathrm{y}$ alto, se manifiesta un nivel significativo del potencial para lograr capital intelectual, si se tiene en cuenta que actualmente no hay un modelo de gestión orientado para tales fines.

Sobre la apreciación del potencial de aportes por parte de los practicantes de ingeniería, realizamos un cuestionario de siete preguntas y entrevistamos a 83 jefes o responsables directos de practicantes de siete especialidades de ingeniería de una universidad.

A continuación, mostramos los datos de las especialidades y dos de los diagramas relevantes para nuestro caso en la figura 2:

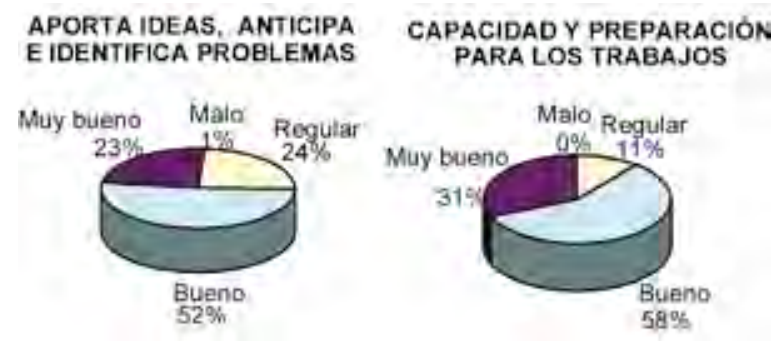

Figura 2: Aporte y capacidad de los practicantes.

Como conclusión general, hay como mínimo un 75\% de apreciación favorable, valorando, por tanto, la capacidad y el conocimiento aplicado por los practicantes. Esto nos muestra un potencial latente en los practicantes como agentes para desarrollar conocimiento y capital intelectual
En cuanto a las necesidades de apoyo por parte de los practicantes de ingeniería, realizamos un cuestionario de siete preguntas a 107 alumnos practicantes de un total de siete especialidades o carreras.

Los datos manifiestan quela necesidad y oportunidad de intervención académica son reales, tal como se aprecia en la figura siguiente, con dos resultados más relevantes:
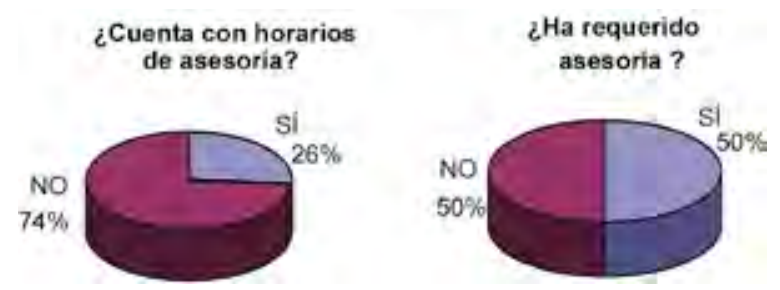

Figura 3: Necesidades concretas de los practicantes.

Respecto a la factibilidad de orientar o conducir prácticas obteniendo monografías o reportes, realizamos un estudio documental en la especialidad de Ingeniería Industrial de una universidad. Se eligió a Ingeniería Industrial por la amplia cantidad de practicantes en relación a las demás carreras, y se contactó a una universidad donde se contaba con registros e informes sostenidos por un periodo mayor a 10 años sobre practicantes, a fin de contar con información suficiente para las comprobaciones.

En este caso, la universidad, llegó a obtener hasta 400 informes en un mismo año.

Pero, se manifestó lo siguiente:

- No hubo consolidación en ningún repositorio;

- Los informes se perdieron y/o destruyeron;

- La información no estaba estructurada;

- No se llegó a una complementación académica entre trabajos, practicantes, y docentes;

- No se realizaron diagnósticos, investigaciones, benchmarking, ni similares; y

- Menos del 5\% de aportes para propuestas de temas de tesis.

Conclusión: Es factible que un practicante, asesorado, desarrolle un informe técnico de una práctica y de manera masiva; hay oportunidades notorias para el aprovechamiento académico, sin embargo, no son abordadas de manera sistemática. 
Sobre el potencial de mejora en las aplicaciones de metodologías o técnicas, elegimos investigar sobre el control estadístico de procesos, dada la amplia aplicación de sus técnicas en diferentes industrias y procesos.

Se conformó un equipo con un docente y dos practicantes que ya habían cursado las asignaturas requeridas para realizar el trabajo de campo. Realizamos visitas a 75 PYMES (16 medianas, 59 pequeñas) del sector confección textil, observando el control aplicado y aplicando un cuestionario de 35 preguntas vinculadas a 20 hipótesis.

Entre los hallazgos, destaca la confirmación del limitado ámbito de aplicación y nivel de rigurosidad y constancia de las técnicas, además de comprobar confusión de conceptos y propósitos de las técnicas.

Como evidencia más relevante mostramos un cuadro sobre causas de la no utilización de técnicas de control de calidad.

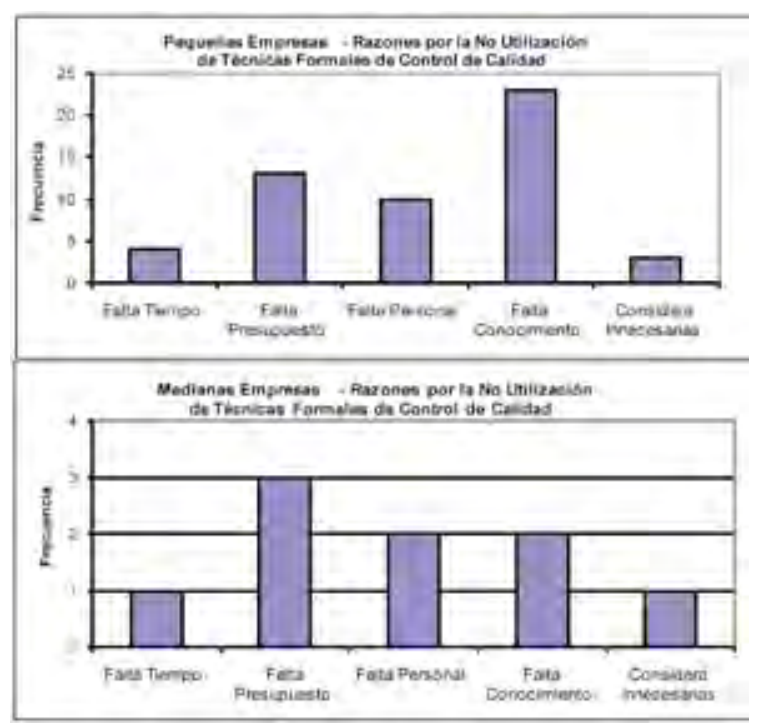

Figura 4: Razones de la no utilización de técnicas formales de control de calidad - Pequeñas y Medianas Empresas.

Se evidencia limitaciones de capacidad de la organización para desarrollar y aplicar el conocimiento [1], además de proporciones similares, excepto en el aspecto "falta de conocimiento", era de esperar que en las pequeñas empresas tenga mayor frecuencia que en las medianas.

Acerca del potencial de investigación aplicada con propuestas concretas en mejora de procesos, realizamos otro piloto conformado por un docente, un ingeniero especialista y 12 practicantes. Se eligió el proceso de confección industrial de prendas de vestir del modelo polo-box (polo camisero con tres botones talla medium), aprovechando los contactos ya obtenidos por el estudio anterior.

Se analizaron los reportes de los practicantes en un grupo de 12 talleres, para seleccionar procesos y operaciones equivalentes para efectos de normalización o estandarización. Es así que se obtuvo un conjunto de 12 operaciones de costura con los tiempos estándares aplicados en cinco talleres. A continuación, mostramos los tres casos más representativos:

\begin{tabular}{|c|c|c|c|}
\hline & EMPRESA 1 & EMPAESA 2 & EMPRESA 3 \\
\hline PEGAR PECHERA & 0.557 & 0.850 & 0.4225 \\
\hline EMBOLSAR PUNTAS CUELLO & 0.570 & 0.680 & 0.8450 \\
\hline UNIR HOMEROS & 0.557 & 0.367 & 0.4000 \\
\hline RECUBRIR HOVBROS & 0.497 & 0.367 & 0.3750 \\
\hline PEGAR CUEUO & 0.004 & 0.460 & 0.5253 \\
\hline ASENTAR CUELLO & 0.721 & 0.830 & 0.9375 \\
\hline PEGAR NANGAS & 0.539 & 2.910 & 0.6516 \\
\hline RECUBRIR SISAS & 0.742 & 0.710 & 0.6549 \\
\hline PREFUAR ETIQUETA & 0.135 & 0.160 & 0.3015 \\
\hline CERRAR COSTADOSAORELLAR PECX & 1471 & 1.160 & 1.0344 \\
\hline OJALAR PECHERA & 0.534 & 0.345 & 0.2939 \\
\hline PEOAR BOTONES & 0.531 & 0.263 & 0.2628 \\
\hline foes Thoos Extandar (Fin) & 8,70 & 600 & 6.595 \\
\hline Rendes por bisoo do Jirs. & B.,03 & $52 \times 10$ & 2.778 \\
\hline
\end{tabular}

Figura 5: Comparativo de tiempos estándares.

Como se observa en los datos de tiempos totales, hay diferencia significativa en un rango de 1,583 minutos por prenda, lo que implica un $23 \%$ del tiempo total del caso intermedio. Tal cifra implica que hay talleres con mayores costos, mayor demora, menor productividad y menor competitividad.

Habrá causas naturales propias de los materiales y puestos, pero para el rango detectado, las causas se hallan vinculadas al desconocimiento $\mathrm{y}$ a las debilidades de las aplicaciones de Ingeniería de métodos y de la Ergonomía.

Dadas las evidencias, pasaremos a presentar nuestra propuesta:

En primer lugar, se requerirá que el modelo se escale y se aplique de manera general a nivel interinstitucional, mediante la participación conjunta de entidades de apoyo, de las universidades con sus unidades académicas y de investigación y de institutos de investigación no universitarios. 
Sobre esta base, desarrollamos nuestra propuesta, estructurada por niveles. Se inicia con el nivel estratégico, pasando al nivel organizacional, siguiendo con el nivel funcional (procesos operativos), para luego plantear un modelo de soporte informático con un planteamiento de las bases de datos y una propuesta de indicadores de medición.

\section{Nivel estratégico}

A este nivel, se propone una planificación del desarrollo del capital intelectual a partir de un estudio estratégico de los sectores industriales que permita determinar prioridades y necesidades sector por sector, para luego establecer los objetivos y planes de investigación, así como determinar los aspectos organizacionales para cumplir los fines proyectados. A continuación se muestra el diagrama del modelo a este nivel:

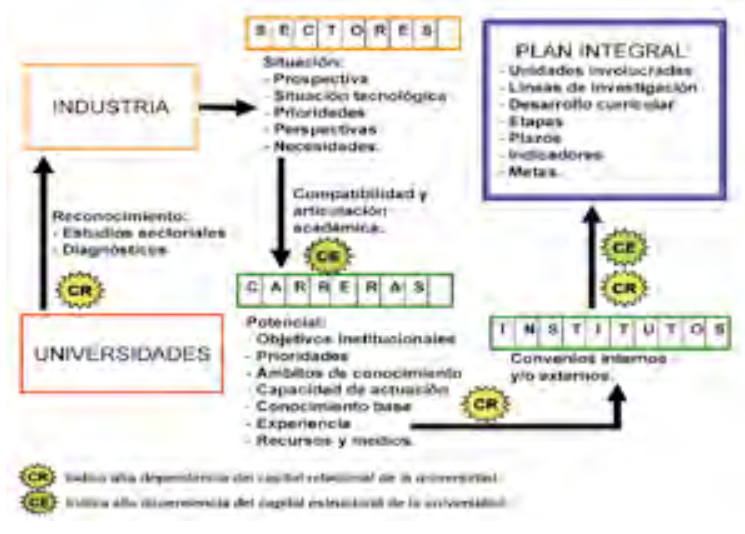

Figura 6: Modelo propuesto: nivel estratégico.

La estrategia consiste en planificar estudios sectoriales para identificar problemas y necesidades actuales de la industria sobre las cuales la Facultad intervendrá como agente de cambio y de mejora.

Esto implica realizar diagnósticos y estudios de sector por sector, lo cual necesita un soporte orgánico, tácticas y condiciones que identificamos mediante los pilotos:

1. Unidad de Desarrollo Industrial (UDI), que dirija procesos del conocimiento y su conversión en capital intelectual;

2. Comités de Desarrollo Industrial (CDI), encargados cada uno de un sector industrial;
3. Equipos de Desarrollo Industrial (EDI), o comunidades de práctica con organización celular y énfasis en el trabajo colectivo, que se integrarán según la necesidad, crecerán y se renovarán en el tiempo, dado que incorporaremos activamente a grupos de estudiantes ciclo a ciclo; y

4. Unidad de Prácticas Preprofesionales, como soporte administrativo y logístico. También apoyará en los contactos y convenios con empresas o industrias (capital relacional).

\section{Nivel de gestión}

Definida la estrategia y la estructura básica que permita lograr los objetivos, la gestión comprende las funciones de planificación, comunicación, organización, coordinación y control.

Por tanto, el modelo a proponer aportará respecto a cada elemento referido, de la siguiente manera:

1. El Decano designa y conforma la UDI;

2. La UDI designa y conforma los CDI, acorde a los sectores y subsectores que priorizará, según planes de desarrollo, anuales o bianuales;

3. Para cada sector nuevo, la CDI convocará, conformará y orientará a los EDI;

4. La UDI interactuará con las unidades ya existentes (facultades, institutos, laboratorios); asimismo, realizará los primeros contactos con representantes de sectores, gremios y empresas o industrias, para concertar el apoyo necesario (capital relacional);

5. La UDI debe incorporar a docentes de la Facultad, además de egresados profesionales y expertos, que brinden soporte académico y consultivo y se incorporen a los EDI;

6. La CDI debe desarrollar, actualizar y/o adaptar las metodologías, técnicas e instrumentos para los diagnósticos que se orienten hacia la mejora e innovación tecnológica en el sector designado; 
7. Cada CDI dirige los diagnósticos y realiza el análisis e informe de cada diagnóstico concluido;

8. Los EDI parten de los diagnósticos, ejecutan los estudios, capturan y convierten el conocimiento tácito en explícito para luego convertirlo en capital intelectual;

9. Los EDI estarán conformados por alumnos practicantes, docentes, investigadores y profesionales especialistas, según se requiera (aprovechando el capital estructural);

10. La unidad de prácticas se encargará de los registros, convenios y controles de los practicantes, sin perjuicio de la supervisión de los CDI.

De esta manera, la UDI va a dirigir los estudios o diagnósticos que cada CDI supervisará y que los EDI ejecutarán sector por sector, determinando un programa de actividades bajo un horizonte anual o bianual, para contar con tiempo suficiente como para obtener información completa y de calidad de significativo número de industrias que pueda sustentar los hallazgos, propuestas y plan de actividades.

De los diagnósticos se obtendrá información sobre:

a. Prospectiva del sector;

b. Impacto económico, social y ambiental;

c. Demandas y exigencias del mercado;

d. Situación tecnológica;

e. Procesos, recursos, productos;

f. Capacidades;

g. Beneficios que disfruta;

h. Carencias y limitaciones;

i. Necesidades; $y$

j. Tendencias, proyecciones e impactos.

Con tal información se determinará el tipo de intervención que planificará cada CDI, estableciendo:

- Propósito, resultados y productos;

- Líneas de investigación vinculadas;

- Aporte a desarrollo curricular;

- Unidades involucradas;
- Especialidades (carreras);

- Etapas y plazos;

- Indicadores y metas para control; y

- Recursos a asignar.

Luego de las definiciones del caso, el CDI, con apoyo de la UDI, contactará y difundirá los planes y proyectos, convocará a participantes y conformará los EDI y las asociaciones que se requieran.

Así se conformarán los EDI para ejecutar los proyectos de investigación aplicada según las necesidades y oportunidades que se presenten. Además, un EDI puede renovar o cambiar sus miembros en el tiempo y dividirse según se requiera.

\section{Nivel organizacional}

Como una organización debe diseñarse para el logro de los objetivos, permitiendo la ejecución integrada de los procesos, se propone una estructura complementaria a las actuales que permita integrar las actividades de investigación aplicada con las demás actividades académicas y de investigación de las especialidades.

Para ello, se requiere:

- Definir estructuras complementarias para la investigación en pregrado;

- Crear la unidad de prácticas pre profesionales para aportar el capital humano; y

- Establecer la UDI, las CDI y las EDI.

En la figura 7 se presenta la propuesta de modelo de estructura orgánica general, para lo cual se ha tomado a una universidad y la facultad como base.

Se muestran las unidades administrativas, académicas y las de investigación principales e involucradas con el proceso de generación de conocimiento y desarrollo de capital intelectual.

Sobre tal soporte estructural se desarrollarán flujos que aprovechen el capital relacional paralos contactos y convenios institucionales, así como el capital estructural, principalmente en lo concerniente al soporte de tipo administrativo. 
Asimismo, en el modelo se muestran los flujos de capital humano que permitirán principalmente la conformación y sostenimiento de los EDI en el tiempo. Aparte de docentes e investigadores propios de las carreras, se considera incorporar a los alumnos de posgrado como un capital más que puede aportar en el nuevo esquema, en paralelo con el desarrollo de sus tesis.

Es importante mantener cierta independencia de las carreras de Ingeniería a través de una unidad de investigación encargada de la gestión general y el concurso de unidades descentralizadas en cada facultad, con la finalidad de asegurar la equidad y la integración de comunidades multidisciplinarias.

Cabe destacar que a este nivel se manifiestan diferencias entre universidades en cuanto a las estructuras orgánicas de sus facultades y las unidades de investigación. Por tanto, a continuación semuestra el diagrama del modelo genérico para vinculación organizacional entre unidades académicas y de investigación:

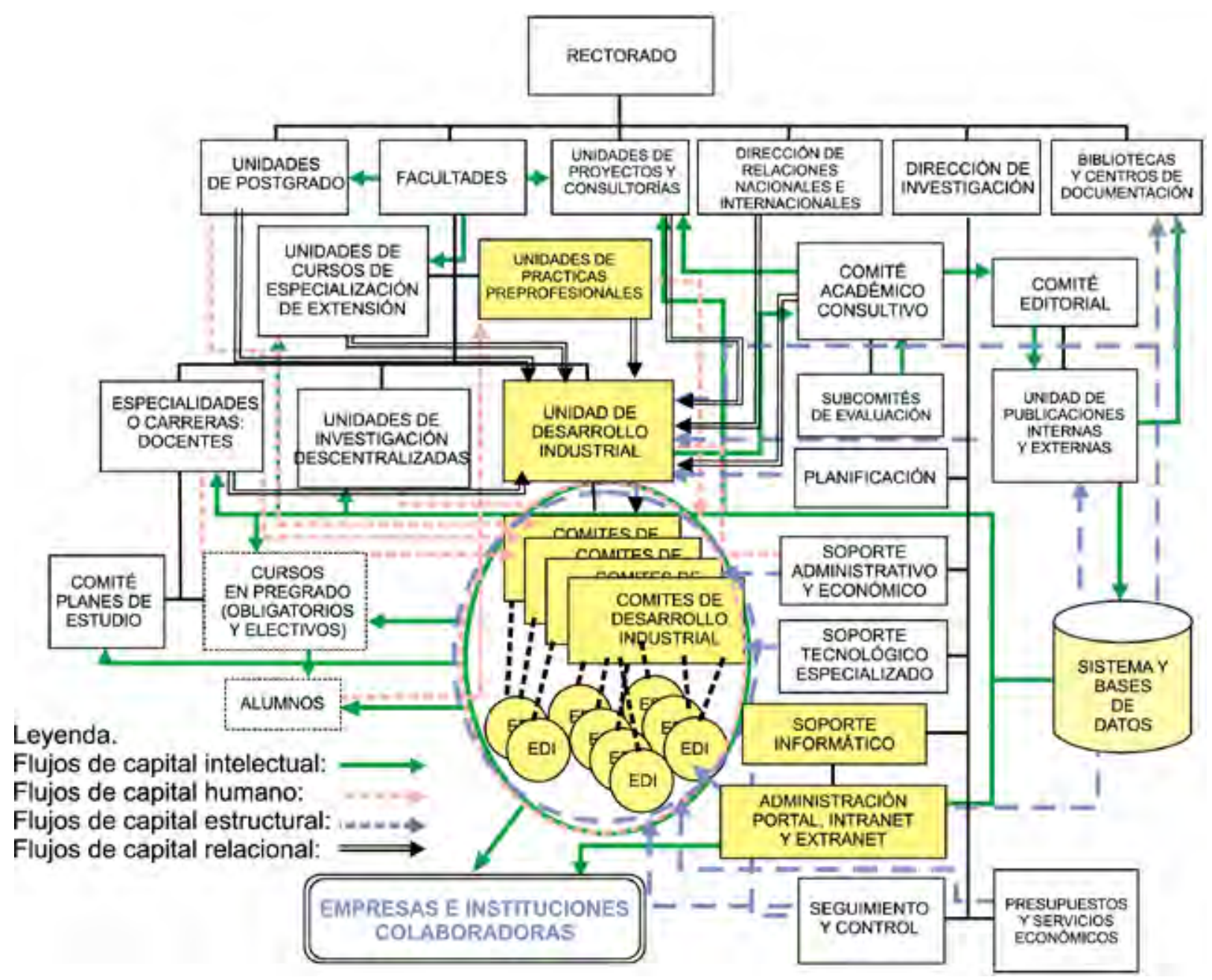

Figura 7. Modelo propuesto, nivel organizacional

\section{Nivel funcional}

Para los EDI, se propone a este nivel la estructura basada en las comunidades de práctica normales con opciones para trabajo virtual, donde participan, de manera no exclusiva:

- Investigadores de la universidad;
- Profesionales egresados;

- Especialistas o consultores;

- Docentes de la carrera; y

- Alumnos de pregrado.

Se recomienda para efectos de una mejor convocatoria de alumnos y mejor aprovechamiento académico, que los EDI se articulen con cursos como: 
- Cursos de fin de carrera;

- Cursos de proyectos de investigación; y

- Cursos de prácticas preprofesionales.

Los cursos deben estar definidos en cuanto a créditos, nivel o ciclo de estudios y requisitos para matricularse y participar.

Como tema para un EDI, consideremos el "Control de calidad en la fabricación de polos camiseros de tejido de punto de algodón para exportación". El EDI se conformaría de la siguiente manera:

- Un orientador o docente de la carrera de ingeniería industrial con experiencia en los cursos de calidad u operaciones;

- Un egresado especialista en control de calidad de procesos de confecciones; $y$

- Cinco alumnos de pregrado, que ya hayan aprobado los cursos de calidad y estadística.

Habrá reuniones previas y visitas en equipo a cada taller para reconocimiento de los factores y condiciones similares y los diferentes.

Los alumnos realizarán el trabajo de campo en cada taller, en equipos de dos personas y se comunicarán entre grupos mediante el sistema; establecerán una red para compartir experiencias y conocimiento con los demás de la comunidad; y serán orientados y asesorados por el docente y el especialista.

Se comunicarán en el tiempo y la distancia, mediante un portal WEB, dentro del cual tendrán acceso a los archivos digitales y también actualizarán y alimentarán las bases de datos y librerías digitales.

Aplicarán la teoría, métodos y técnicas propias de los ámbitosdeconocimientoinvolucrados, intercambiarán conocimientos adquiridos, reconocerán diferencias, unificarán criterios y concluirán obteniendo un modelo de proceso de control de calidad capaz de ser aplicado a cualquier taller que desarrolle el mismo tipo de producto, bajo ciertas condiciones operativas necesarias según el caso.

A continuación, presentamos el modelo simplificado del proceso. Mostramos en un nivel mayor las etapas para un estudio sectorial con participación de un CDI $y$, a un nivel menor, las etapas que corresponden a los proyectos de investigación con participación de los EDI:

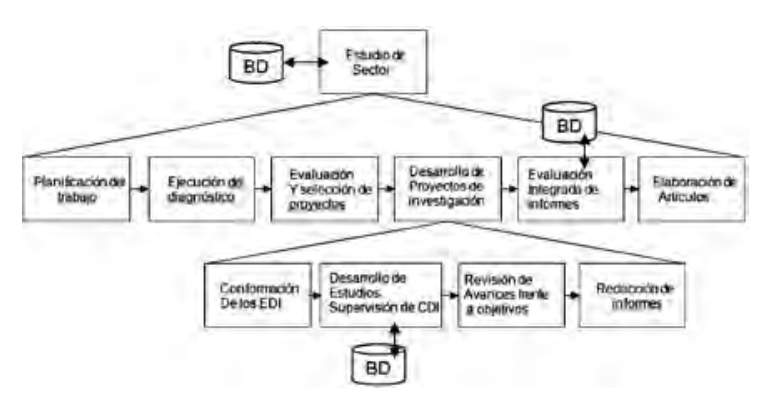

Figura 8: Modelo propuesto para el proceso de generación de CI.

\section{Soporte informático}

Será fundamental por la necesidad de relacionar bajo un entorno colaborativo a diversas personas en el tiempo y el espacio, así como por el soporte para el ordenamiento, resguardo, manejo y difusión de la información.

A continuación se muestra un diagrama conceptual de los componentes del modelo a este nivel:

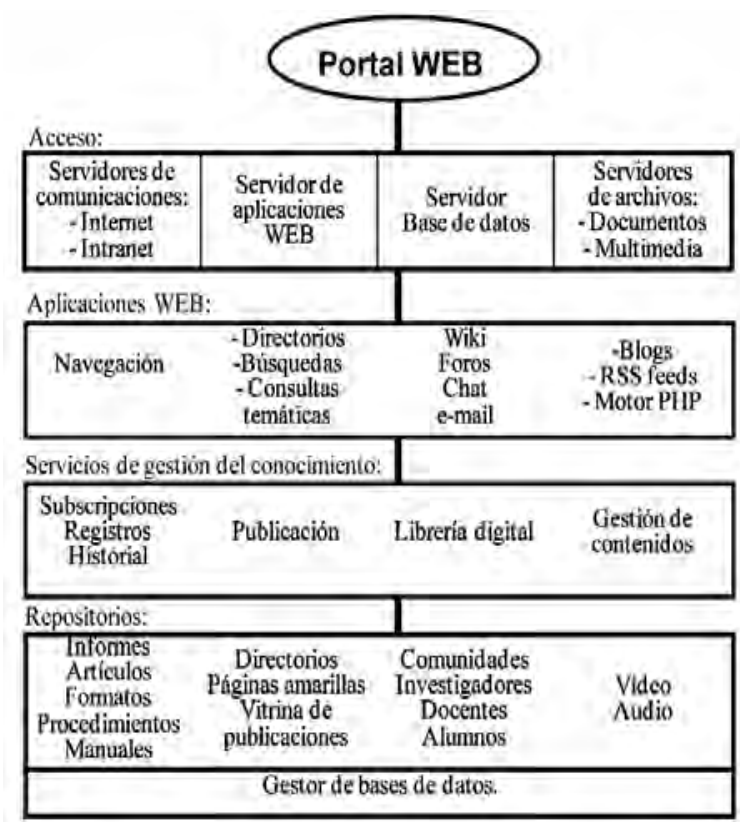

Figura 9: Modelo propuesto, soporte informático.

En lo que corresponde a la información básica a manejar, identificamos 16 tablas que estructurarán las bases de datos, destacando:

- Planes y proyectos;

- CDI y EDI;

- Instituciones de apoyo y empresas;

- Códigos CIIU y UNESCO, para clasificación; 
- Temas (ámbitos de conocimiento);

- Alumnos, egresados, docentes, especialistas;

- Informes: prácticas, estudios realizados; y

- Publicaciones a difundir.

En cuanto a la información sobre industrias y procesos, las bases de datos serán complejas y diferentes según la especialidad o carrera. Como se trata del ámbito industrial, es importante para el diseño la definición de taxonomías para clasificar la información según las siguientes variables generales:

- Proceso: tipo, operaciones, secuencia, tiempos, indicadores de eficiencia, eficacia, utilización, calidad, productividad;

- Producto: tipo, subtipo, especificaciones;

- Tecnología básica: tipo;

- Material: clases, sustitutos, especificaciones;

- Maquinaria y equipo: tecnologías específicas, alternativas; $y$

- Aplicaciones de ingeniería.

Para esto se requerirá de:

- Una infraestructura propia, diseño de red, con servidor dedicado;

- Portal web para el acceso de las comunidades; y

- Bases de datos estructurados para brindar el soporte requerido para efectos de gestión y difusión del conocimiento y capital intelectual a generar.

Dada la complejidad y diversidad de procesos, se ha comprobado dificultades al trabajar con información no estructurada, así como la necesidad de archivos multimedia, con la necesaria aplicación de ontologías para optimizar las búsquedas. Esto se deberá desarrollar a medida que se recabe información de los EDI.

Finalmente, tenemos los principales indicadores para el modelo:

a) Del capital estructural:

- Cantidad de publicaciones por año y por carrera;

- Cantidad de estudios realizados por sector;

- Cobertura de procesos investigados por sector;
- Mejoras a los planes de estudio;

- Técnicas y metodologías de ingeniería aplicadas; e

- Incremento del acervo de experiencias por las aplicaciones realizadas de las técnicas de ingeniería.

b) Del capital humano:

- Incremento anual de comunidades de práctica;

- Grado de permanencia de actividad de las comunidades conformadas;

- Investigadores en actividad dentro del sistema; $\mathrm{y}$

- Alumnos y ex alumnos en actividad dentro del sistema.

c) Del capital relacional:

- Convenios de cooperación académica;

- Convenios de cooperación universidadempresa;

- Convenios de cooperación con agremiaciones e instituciones industriales; $y$

- Convenioscon instituciones de apoyo económico para la investigación (como los fondos BID).

d) De tipo estratégico:

- Resultados en los indicadores de productividad de los procesos intervenidos en las empresas, a través de implantaciones por los EDI;

- Cobertura de sectores por los planes de investigación, y proyectos ejecutados efectivamente;

- Cantidad de proyectos culminados con éxito sobre el total ejecutado, por sector y carrera; y

- Cobertura y cantidad de mapas tecnológicos levantados por sector y subsector.

\section{CONCLUSIONES}

El modelo propuesto aportará a cada función de la gestión del capital intelectual.

El modelo propuesto facilita la obtención de capital intelectual (CI) producido por las EDI con un potencial de generar valor para la especialidad involucrada, la universidad, las empresas, el sector, y la sociedad, mediante: 
- Conocimiento explícito convertido en CI al ser aplicado en las industrias;

- Acceso al CI efectivo y democrático;

- Incremento de la capacidad de absorción del conocimiento en PYMES;

- Incremento de la productividad de los procesos e industrias, al implantarse el CI;

- Progresiva mejora de sectores industriales, al extenderse las actividades;

- Desarrollo de nuevos cursos electivos y casos de estudio para los cursos vigentes;

- La información directa y útil para actualizar y mejorar los planes de estudio de la carrera;

- El desarrollo de cursos de extensión;

- Oportunidad para proyectos de consultoría; y

- Oportunidad para investigaciones básicas sobre aspectos específicos detectados por los EDI.

\section{AGRADECIMIENTOS}

Agradecemos a todas las Facultades de Ingeniería de las universidades que apoyaron en las encuestas y a los docentes e investigadores que apoyaron en las entrevistas.

\section{REFERENCIAS}

[1] W. M. Cohen, D. Levinthal, Absorptive Capacity: A New Perspective On Learning And Inno. Administrative Science Quarterly; ABI/INFORM Global, 1990.

[2] E. Bueno et al., Gestión del Conocimiento en Universidades y Organismos Públicos de Investigación. Universidad Autónoma de Madrid, 2003.

[3] L. García, Estrategias de Gestión para la Capitalización del Conocimiento en el contexto de la Relación Universidad Sector Productivo, en la revista Educere; año/vol 8, Número 027: Universidad de los Andes, Mérida, Venezuela, 2004.

[4] L. Warren, Creating the Entrepreneurial University, Compilado en "Encyclopedia of Communities of Practice in Information and Knowledge Management," (2006), 108-114.

[5] B. Ditzel, Desarrollo de un modelo de gestión del conocimiento para un Departamento universitario, Campus Tecnológico de la Universidad de Navarra, 2005.

E-mail:formach@pucp.edu.pe erikpapa@gmail.com 